\title{
Ritmo idioventricular asociado a cuadro vagal
}

\author{
Idioventricular rate asociated to vagal situation
}

\author{
Leydimar Anmad-Shihadeh*, María Ramos-López, Luis Álvarez-Acosta, Diego Valdivia-Miranda, \\ María Facenda-Lorenzo y Julio Hernández-Afonso \\ Servicio de Cardiología, Hospital Universitario Nuestra Señora de la Candelaria, Santa Cruz de Tenerife, España
}

Presentamos el caso de una mujer de 73 años, ingresada en nuestro Servicio tras presentar síncope y un electrocardiograma (ECG) con una taquicardia de QRS ancho. Refería antecedentes de alergia al ácido risedrónico, hipertensión arterial y dislipemia. Sin antecedentes personales ni familiares de cardiopatía. Tomaba como tratamiento enalapril e hidroclorotiazida. La paciente acudió a Urgencias por presentar síncope sin pródromos caminando por su casa, después de haberse levantado de la cama y miccionar. Tras recuperarse del síncope, presentó vómitos y mareo intenso. A la anamnesis dirigida refiere episodios presincopales y sincopales desde la infancia, siempre en el contexto de estrés, visiones desagradables o dolor. A su llegada a Urgencias está nauseosa, con tensión arterial de $145 / 71 \mathrm{mmHg}$, buen estado general y una exploración física anodina, salvo taquicardia regular a $120 \mathrm{lpm}$. En la primera analítica de sangre tiene una creatinina de $1.04 \mathrm{mg} / \mathrm{dl}$ (normal), iones normales, CPK $217 \mathrm{U} / \mathrm{l}$ (normal), Tropl US 0,04 ng/ml (normal), hemograma y coagulación normal. En el ECG de Urgencias se observa taquicardia regular de QRS ancho a $120 \mathrm{lpm}$ con QRS con morfología de BCRDHH y eje hiperdesviado a la derecha sin concordancia auriculoventricular en los tres primeros latidos (Fig. 1A, flechas en rojo) y captura auricular a partir del cuarto latido (flechas en azul con $P$ negativa inferior), terminando en $\mathrm{VA}$, antes de pasar a ritmo sinusal.

Aunque el perfil del síncope es vasovagal y tiene características de neuromediado, se plantea como primera posibilidad diagnóstica que se trate de un síncope de causa arrítmica; debido a los hallazgos electrocardográficos e implementando herramientas de estratificación de riesgo de mortalidad descritas como OESIL (cercano al $20 \%$ en nuestra paciente) quedó ingresada para estudio. Durante su estancia en planta permaneció monitorizada, manteniendo ritmo sinusal en todo momento. Se realizó: ecocardiograma, que resultó rigurosamente normal; y prueba de esfuerzo que duró siete minutos, con protocolo Bruce, alcanzando el $100 \%$ de la frecuencia cardíaca máxima teórica, sin arritmias y negativa para isquemia. Decidimos no realizar coronariografía por baja sospecha de enfermedad coronaria, pero sí estudio electrofisiológico. Al inicio del estudio, tras la punción venosa, la paciente presenta cuadro vagal que responde a atropina y posteriormente presenta ritmo idioventricular compitiendo con el nodo sinusal (Fig. 1B); vemos de nuevo una taquicardia de QRS ancho con BRDHH y eje hiperdesviado a la derecha, con ondas $\mathrm{P}$ positivas en II que interpretamos como

\section{Correspondencia:}

*Leydimar Anmad-Shihadeh

Servicio de Cardiología

Hospital Universitario Nuestra Señora de Candelaria

Ctra. del Rosario, 145

Fecha de recepción: 19-05-2018

Fecha de aceptación: 02-10-2018

DOI: 10.24875/ACM.M19000017
Disponible en internet: 19-03-2019 Arch Cardiol Mex. 2019;89(1):96-98 www.archivoscardiologia.com 


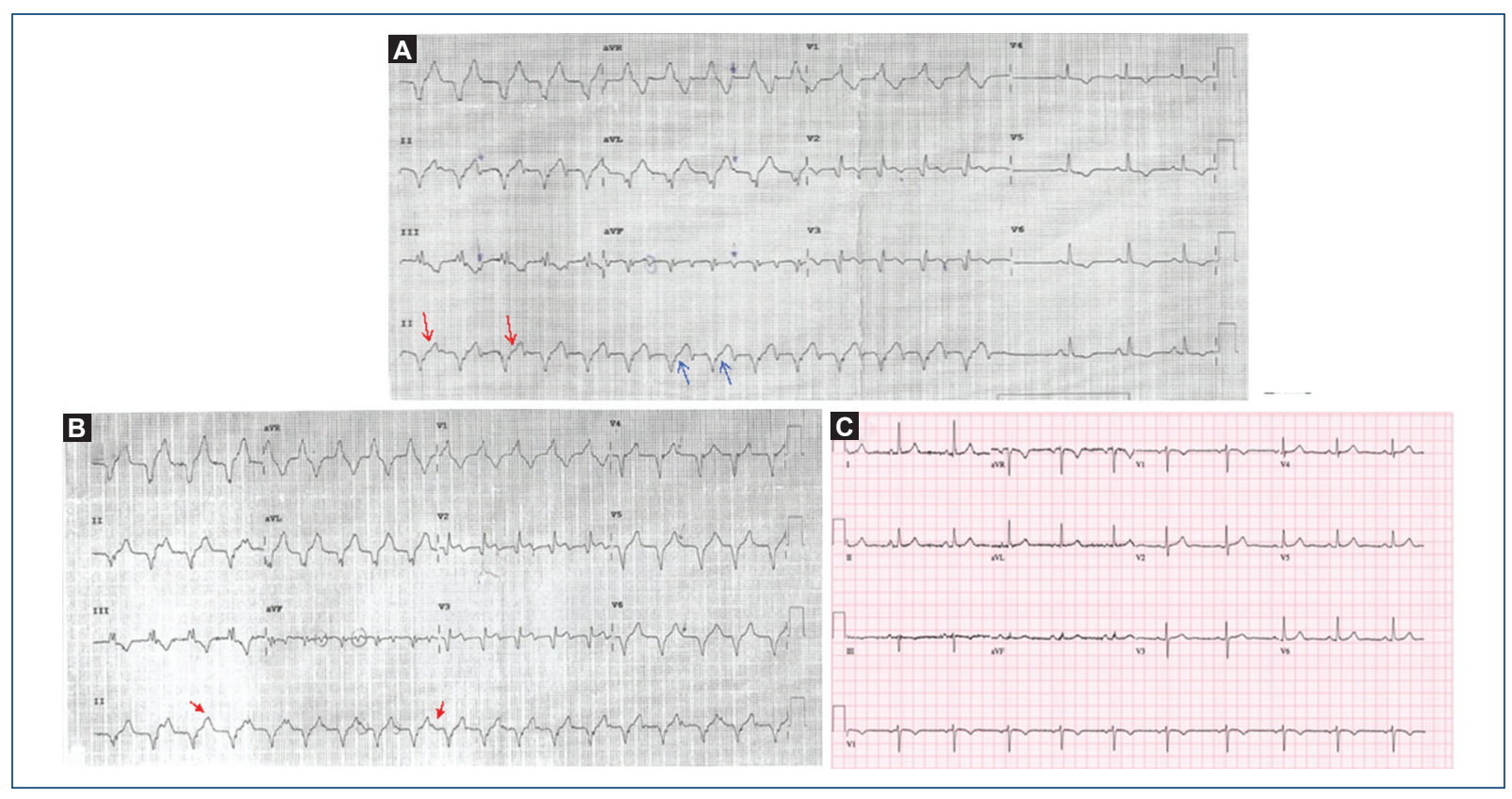

Figura 1. A: taquicardia regular de QRS ancho a $120 \mathrm{lpm}$ con QRS con morfología de BCRDHH y eje hiperdesviado a la derecha sin concordancia auriculoventricular, en los tres primeros latidos y concordancia VA a partir del $4 .^{\circ}$ latido. Terminando en VA, antes de pasar a ritmo sinusal. B: taquicardia regular de QRS ancho a $120 \mathrm{lpm}$ con ORS con morfología de BCRDHH y eje hiperdesviado a la derecha, sin concordancia auriculoventricular (ver flechas). C: ritmo sinusal, eje normal.

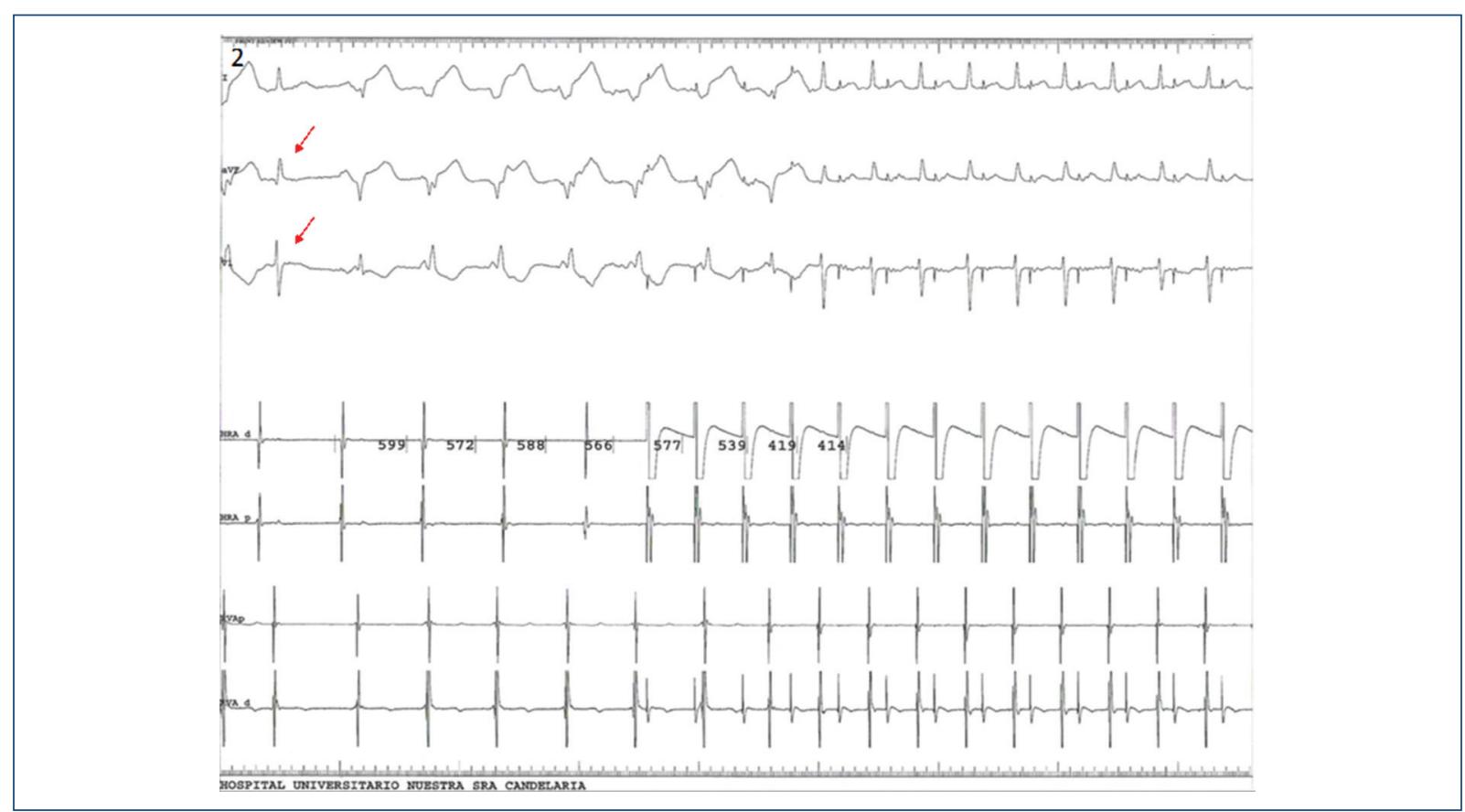

Figura 2. Registro del estudio electrofisiológico, donde se evidencia disociación auriculoventricular. Primer latido sinusal (flechas rojas), tras el cual se inicia el RIVA; en los registros, tanto de los catéteres posicionados en aurícula derecha alta y baja, como en ventrículo derecho, se evidencia que no hay concordancia ventriculoauricular. Posteriormente en el canal auricular se inicia estimulación con trenes de 522 hasta 414 ms, se corta el RIVA y no se evidencia aberrancia de la conducción. 
ritmo RIVA con actividad auricular sinusal disociada (ver flechas). Se introducen los catéteres en $A D$ y ápex de VD objetivándose disociación VA. Punto de Wenckebach a $290 \mathrm{~ms}$ (Fig. 2). El ritmo se mantuvo durante el estudio en forma de salvas autolimitadas, con la paciente asintomática. Fue dada de alta con el diagnóstico de ritmo idioventricular secundario a síncope vasovagal, sin tratamiento farmacológico.

Como sabemos, el RIVA se caracteriza electrocardiográficamente por un ritmo de complejo QRS ancho con al menos tres complejos ventriculares consecutivos a una velocidad típica de 40 a $120 \mathrm{lpm}$, es decir, más rápido que la frecuencia ventricular intrínseca, pero más lento que la taquicardia ventricular.

Este ritmo se presenta frecuentemente en presencia de cardiopatía, como suele ocurrir durante la reperfusión coronaria en el infarto agudo de miocardio (IAM). Sin embargo, también puede suponer un hallazgo casual y ha sido documentado en ausencia de cardiopatía estructural. Un mecanismo ampliamente aceptado para RIVA, al menos en el IAM, es la automaticidad mejorada de las fibras de His-Purkinje, que da como resultado un ritmo ventricular con una frecuencia mayor que la del marcapasos sinusal (cuya frecuencia de descarga pudiera estar enlentecida por aumento del tono vagal y disminución del tono simpático). El estrés emocional o el dolor y el estrés ortostático ocasionan una cascada de eventos cuya fisiopatología se explica por una resistencia periférica baja o inadecuada debida a una actividad refleja inapropiada, apareciendo vasodilatación y bradicardia por aumento del tono vagal; en este contexto, un ritmo ventricular automático favorecido por estimulación de mecanorreceptores podría dar lugar a RIVA'.

La sintomatología que presentaba nuestra paciente, junto con su historial de síncopes y las circunstancias en que se producían (estrés, cambios posturales y dolor) sugieren un síncope neuromediado y, como sabemos, este es un diagnóstico la mayor parte de las veces clínico. En nuestro caso, la aparición de esta arritmia relacionada con estas circunstancias indica una asociación íntima entre RIVA y el desequilibrio autonómico producido?2.

La mayoría de los casos de RIVA son hemodinámicamente estables, autolimitados y no requieren tratamiento. Aunque no implica mal pronóstico, su aparición no asociada a IAM sí que nos obliga a un estudio más o menos exhaustivo para descartar patología subyacente ${ }^{3}$.

\section{Conflicto de intereses}

Los autores declaran no tener conflicto de intereses ni haber recibido financiación.

\section{Responsabilidades éticas}

\section{Protección de personas y animales}

Los autores declaran que para esta investigación no se han realizado experimentos en seres humanos ni en animales.

\section{Confidencialidad de los datos}

Los autores declaran que han seguido los protocolos de su centro de trabajo sobre la publicación de datos de pacientes.

\section{Derecho a la privacidad y consentimiento informado}

Los autores declaran que en este artículo no aparecen datos de pacientes.

\section{Bibliografía}

1. Muniyappa A, Goldschlager N. Wide complex ventricular rhythm in a patient after collapse. JAMA Intern Med. 2017;177(6):872-73.

2. Kataoka $\mathrm{H}$. Atrioventricular dissociation as a cause of syncope determined by head-up tilt test. Pacing Clin Electrophysiol. 1999;22(12):1835-7.

3. Wilde AA, Wieling W. Vasovagal syncope or ventricular fibrillation. Your diagnosis better be accurate. Clin Auton Res. 2007;17(4):203-5. 